\title{
Un estudio sobre la diferencia de nivel de competencia lingüística entre alumnos pertenecientes a centros bilingües y no bilingües en la comunidad autónoma de Castilla-La Mancha (España)
}

\section{A study about the differences in the English language level of students inmmersed in bilingual and non-bilingual programmes in Castilla-La Mancha (Spain)}

\author{
ARSENIO JESÚS MOYA GUIJARRO \\ MARÍA BEGOÑA RUIZ CORDERO \\ Universidad de Castilla-La Mancha, España. \\ Correo electrónico: Arsenio.MGuijarro@uclm.es \\ Universidad de Castilla-La Mancha, España. \\ Correo electrónico: Mariabegona.Ruiz@uclm.es
}

\begin{abstract}
En los últimos años ha habido un cambio positivo en la percepción sobre el bilingüismo que se refleja en un incremento considerable en la demanda de educación bilingüe entre muchos ciudadanos. Así, el bilingüismo constituye un elemento clave de oportunidades que cualquier miembro de nuestra sociedad puede aprovechar independientemente de cual sea su situación económica familiar (Salis 2002: 43; García 2009: 11). Por este motivo, cada vez más alumnos se incorporan a programas bilingües, pero ¿funcionan?, ¿se obtienen buenos resultados? El trabajo que aquí presentamos pretende analizar la diferencia de nivel competencial de lengua inglesa entre los alumnos de $4^{\circ}$ curso de Educación Secundaria Obligatoria pertenecientes a programas bilingües y los que siguen enseñanzas convencionales en la región española de Castilla-La Mancha. Con la información obtenida en nuestro análisis podremos comprobar si los programas bilingües mejoran o no la competencia comunicativa en lengua inglesa de los alumnos inmersos en ellos y, a su vez, establecer una comparación entre los resultados hallados dentro del programa bilingüe de Castilla-La Mancha y los programas desarrollados en otras comunidades autónomas.
\end{abstract}

Palabras clave: Bilingüismo, Educación Secundaria, nivel de lengua inglesa, Castilla-La Mancha.

In recent years there has been a positive change in the perception about bilingualism reflected in the increasing number of citizens demanding bilingual education. In this way, bilingualism constitutes 
a key element of opportunities (Salis 2002: 43) for any member of society regardless of their economic situation (García 2009: 11). For this reason, the number of students joining bilingual programmes is increasing but, do they work? do they produce positive results? This research aims to analyse the differences in the English level between 4th year secondary school students belonging to bilingual and non-bilingual programmes in the Spanish region of Castilla-La Mancha. With the obtained information in our analysis we can determine if the bilingual programmes increase the communicative competence in English language of the students immersed in them. In addition, we will establish a comparison between the results acquired in the bilingual programme of Castilla-La Mancha and other programmes developed in different autonomous communities.

Key words: Bilingualism, Secondary Education, English language level, Castilla-La Mancha.

\section{INTRODUCCIÓN}

Los programas bilingües en la actualidad utilizan la metodología de "Aprendizaje Integrado de Contenidos y Lenguas Extranjeras" (a partir de ahora AICLE), denominada en inglés "Content and Language Integrated Learning" (CLIL). La implantación de asignaturas en lengua extranjera a través de esta metodología, fundada por Mohan en 1986, se está extendiendo cada vez más rápido en España y ha ganado gran relevancia en los últimos años debido, principalmente, a su eficacia como medio para aprender otro idioma de una forma natural. Este enfoque pedagógico se centra más en el desarrollo de las destrezas generales y en el contenido de las materias académicas que en el idioma en sí mismo. Autores como Brinton et al. (1989), o Frufauf, Coyle \& Christ (1996), junto con Marsh \& Langé (1999), apoyan la puesta en marcha de esta metodología y destacan su flexibilidad para introducirla también en contextos no bilingües (Fernández Fontecha, 2001: 218). Más recientemente, contamos con las aportaciones de Mehisto, Marsh \& Frigols (2008), de Coyle, Hood \& Marsh (2010) y de Marsh (2013: 11), los cuales destacan el poder innovador de la metodología AICLE, aunque ciertamente su efectividad sea variable en función del contexto en el que se desarrolle.

En la actualidad, hay un gran número de estudios sobre la evaluación del uso de la metodología AICLE en la adquisición de la competencia comunicativa en lengua extranjera, a veces con resultados beneficiosos. Otras, por el contrario, con resultados menos positivos (Admiraal et al. 2006; Meshisto et al. 2008; Lagasabaster 2008; Jiménez Catalán \& Ruiz de Zarobe 2009; Whittaker \& LLinares 2009; San Isidro 2009, 2010). Partiendo de los resultados de estas investigaciones, en las que parece evidente que los programas bilingües pueden llegar a lograr que se incremente el nivel de lengua extranjera en el alumnado, el objetivo de nuestro estudio es demostrar si los alumnos de $4^{\circ}$ curso de ESO (Educación Secundaria Obligatoria), pertenecientes a programas bilingües ofertados por centros educativos públicos en la comunidad autónoma de Castilla-La Mancha, obtienen mejores resultados globales en la evaluación de la competencia comunicativa en lengua inglesa que los alumnos involucrados en contextos no bilingües. 
Sin embargo, antes de comenzar el estudio se nos plantea la siguiente cuestión: ¿hasta qué punto los alumnos pertenecientes a programas bilingües y el resto de alumnos (que siguen enseñanzas convencionales de lengua inglesa) constituyen grupos equiparables en Educación Secundaria? A este respecto, existen investigaciones que afirman que los estudiantes que acceden al programa bilingüe en Educación Secundaria registran, de antemano, mejores resultados que sus compañeros en comunicación lingüística en inglés (Grisaleńa, Campo \& Alonso, 2009).

A su vez, Bruton (2011) detecta, como aspecto problemático a la hora de valorar los resultados obtenidos en su estudio sobre enseñanza bilingüe, el hecho de que el alumnado acceda a los programas bilingües de manera voluntaria. Esta circunstancia podría conllevar una ventaja inicial tanto en el nivel de inglés como en la motivación de dicho alumnado. El mismo inconveniente aparece en nuestro estudio, debido a que, en Castilla-La Mancha, el procedimiento de admisión a los programas bilingües en Educación Secundaria es voluntario y sólo los alumnos que se ven preparados (que han obtenido buenas calificaciones en lengua inglesa en enseñanzas primarias) o que están muy motivados con respecto al idioma extranjero, deciden comenzar sus estudios de enseñanza secundaria en estos programas lingüísticos. Este factor es un motivo por el que podemos prever que los resultados obtenidos en el nivel de idioma por alumnos de centros con programas bilingües serán mucho más elevados que los conseguidos por estudiantes que no cursan este tipo de programas.

Este artículo queda estructurado en las siguientes secciones. En primer lugar, se muestra el origen de los programas bilingües en la comunidad castellano-manchega. En segundo lugar se presenta el trabajo de investigación en el que se detalla el tipo de alumnado que ha participado, la prueba a la que han sido sometidos los estudiantes y el lugar en el que se ha realizado la misma. En la tercera sección de este trabajo se exponen y analizan los resultados alcanzados en la prueba realizada. Además, para completar este estudio, se comparan los datos conseguidos en Castilla-La Mancha con los de otras comunidades autónomas españolas, más avanzadas en programas bilingües y en las que ya se han llevado a cabo investigaciones similares. Finalmente, para terminar el artículo, se exponen las conclusiones alcanzadas.

\section{ORIGEN DE LOS PROGRAMAS BILINGÜES}

El proyecto bilingüe de la comunidad autónoma de Castilla-La Mancha comenzó a desarrollarse en el año 1996 con el programa British-MEC y las Secciones Hispano-Francesas. Posteriormente, en el año 2005 y como respuesta a las recomendaciones e iniciativas europeas acerca de la necesidad del aprendizaje de lenguas extranjeras y al impulso comunitario de los programas AICLE, nació el plan bilingüe denominado Secciones Europeas. A través de la orden de 07/02/2005 se crearon Secciones Europeas en 36 centros de Castilla-La Mancha: 18 colegios y 18 institutos. En 20 centros (10 colegios y 10 institutos) el idioma que se implantó en la Sección fue el francés y en 16 centros (8 colegios y 8 institutos) la lengua de instrucción fue el inglés, lengua cuyo nivel vamos a medir en este estudio. 
Es importante mencionar que la expansión de las enseñanzas bilingües en España tiene lugar en torno a mediados de la primera década del año 2000. Por ello, la creación en 2005 de las Secciones Europeas en Castilla-La Mancha se encuentra en consonancia con el nacimiento de otros programas en otras regiones monolingües españolas como Andalucía, con su "Plan de fomento del plurilingüismo" (BOJA 05/04/2005), Castilla León, que inició sus Secciones Bilingües mediante la Orden EDU 6/2006 de 4 de enero, o las comunidades autónomas de Madrid o Extremadura, que los desarrollaron en el curso 2004/2005.

El sistema de las Secciones Europeas en Castilla-La Mancha, creado por la orden de 2005, define las Secciones Europeas en su artículo primero como "aquellos centros docentes que incorporan al currículo la enseñanza de áreas no lingüísticas en primera (inglés) o segunda lengua extranjera (francés)". Para poderlo llevar a cabo, los centros deben seleccionar dos materias que han de impartirse en el idioma de la Sección. Además, anualmente se publican convocatorias para que nuevos centros entren a formar parte de estos programas bilingües $y$, a su vez, se van introduciendo nuevas mejoras y diversos cambios que corrigen los problemas que se van detectando en los programas bilingües antiguos ${ }^{1}$. En la actualidad, debido a la orden de 16/06/2014, los programas bilingües de Castilla-La Mancha llamados Secciones Europeas reciben el nombre de Programas Lingüísticos. Desde los inicios del programa bilingüe en 2005 hasta la actualidad se ve un aumento progresivo en el número de Programas Lingüísticos incorporados en educación primaria y secundaria. En total, en el curso académico 2015/2016 se ha contado con 605 Programas Lingüísticos, distribuidos en centros de educación infantil y primaria y en centros de educación secundaria. En la Figura 1 podemos apreciar cómo se han ido incorporando los programas a lo largo de los años y como, por ejemplo, el curso académico 2014/2015, en el que realizamos este estudio, fue en el que se produjeron más incorporaciones.

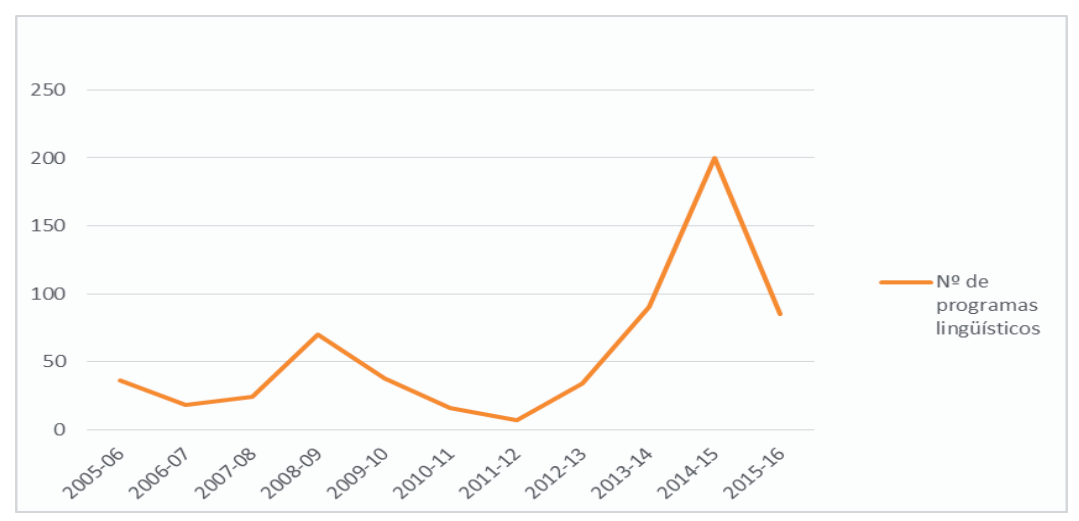

Figura no 1: Incorporación de programas bilingües en Castilla-La Mancha según el curso académico.

\footnotetext{
${ }^{1}$ Orden de 07/02/2005 de la Consejería de Educación y Ciencia por la que se crea el programa de Secciones Europeas en centros públicos de educación infantil, primaria y secundaria de la Comunidad Autónoma de Castilla-La Mancha.
} 
En cuanto a la lengua extranjera implantada en estos 605 Programas Lingüísticos, podemos señalar que hay 563 programas de inglés, 39 de francés, 1 de italiano y 2 de alemán. En la figura 2 que se presenta a continuación se muestra cómo el idioma que predomina es el inglés, seguido de la lengua francesa, y cómo las secciones de italiano y de alemán no llegan a representar el 1\% del total de los programas desarrollados en la región castellano-manchega.

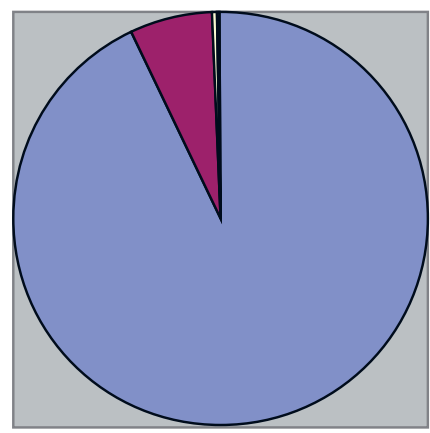

\section{$\square$ 93\% Inglés \\ $\square 6,4 \%$ Francés \\ $\square 0,16 \%$ Italiano \\ $\square 0,33 \%$ Alemán}

Figura no 2: Porcentaje de Programas Lingüísticos por idioma. Curso 2015/2016.

\section{El estudio}

Una vez expuestos el objetivo principal y la hipótesis de partida que genera este trabajo de investigación, con el fin de poder medir y comparar el nivel de lengua inglesa de los alumnos que cursan enseñanzas en centros públicos de Educación Secundaria Obligatoria (a partir de ahora ESO), con y sin Programa Lingüístico en Castilla-La Mancha, describiremos algunos aspectos metodológicos de interés. En primer lugar, se hará referencia a las características del alumnado que ha participado en este estudio. En segundo lugar, vamos a detallar el modo en el que se han seleccionado los centros educativos de los que procede el alumnado evaluado. Seguidamente, se describirá el examen que han realizado los estudiantes de $4^{\circ}$ curso de ESO, tanto de centros bilingües como no bilingües. A su vez, con la información que nos proporcione el análisis comprobaremos si los programas bilingües mejoran o no el nivel de lengua inglesa de los alumnos inmersos en ellos y, a su vez, compararemos su nivel de idioma con el de los alumnos que siguen enseñanzas convencionales.

\subsection{Alumnado participante}

En estas pruebas han participado 201 alumnos de $4^{\circ}$ curso de ESO con edades comprendidas entre los 15 y 16 ańos. 73 estudiantes son alumnos de centros educativos con Programa Lingüístico y 128 son alumnos pertenecientes a centros que no tienen 
Programa Lingüístico implantado en su centro de Educación Secundaria ${ }^{2}$. El nivel cultural y socioeconómico de los alumnos es variable, incluyéndose alumnado de la zona rural y urbana. Todo el alumnado participante ha realizado la prueba de forma anónima, especificando exclusivamente su número de clase (dato que ha utilizado su centro para comunicar el nivel de idioma a sus familiares) y su sexo. A su vez, todos los participantes han realizado el test sin saber que se iba a realizar una investigación con los resultados obtenidos. De este modo, los estudiantes han llevado a cabo la prueba de forma relajada y no se han visto condicionados por ningún aspecto concreto relacionado con esta investigación.

En cuanto a los conocimientos previos en lengua inglesa que poseen los alumnos testados, debemos explicar que todos los estudiantes de centros con Programa Lingǘstico en Educación Secundaria proceden de colegios públicos de Educación Primaria en los que se han llevado a cabo proyectos bilingües desde, al menos, el tercer curso de primaria. Por tanto, estos alumnos, considerados bilingües, llevan, como mínimo, 8 años expuestos tres veces más a la lengua inglesa que sus compañeros pertenecientes a centros sin programas bilingües. Esto se debe a que, aparte de la asignatura de inglés, los estudiantes de programas bilingües han cursado al menos dos asignaturas no lingüísticas en lengua inglesa a través de la metodología AICLE. A su vez, según comentan sus profesores, muchos de estos adolescentes reciben enseñanzas privadas en inglés fuera del horario escolar.

Sin embargo, para la mayoría del alumnado que procede de centros no bilingües, la presencia del inglés fuera del contexto escolar es mínima. No obstante, es necesario mencionar que así todo el alumnado cuenta con recursos como internet en sus casas a través del cual pueden estar en contacto permanente con la lengua extranjera. Estos aspectos han sido constatados por el profesorado de los centros con los que hemos estado en contacto permanente.

\subsection{Selección de centros educativos}

El número de institutos públicos elegido para la realización de las pruebas de nivel de lengua inglesa que accedió a participar en este trabajo ha sido ocho. Cuatro de ellos cuentan con el Programa Lingüístico y, por ello, a partir de ahora, los denominaremos centros bilingües. Los otros cuatro institutos siguen enseñanzas convencionales en lengua extranjera y nos referiremos a ellos como centros no bilingües. En referencia a la etapa educativa, seleccionamos los alumnos que estaban cursando Educación Secundaria, en concreto $4^{\circ}$ curso de $\mathrm{ESO}$, porque queríamos comprobar el nivel de idioma que alcanzan los alumnos al acabar la etapa de enseñanza obligatoria ${ }^{3}$.

\footnotetext{
${ }^{2}$ Como el número de alumnos evaluados en centros educativos con y sin Programa Lingüístico no coincide, posteriormente mostraremos el estudio estadístico realizado para comprobar que los datos obtenidos son estadísticamente significativos.

${ }^{3}$ En el Sistema Educativo Español la enseñanza básica obligatoria tiene dos etapas: primaria y secundaria. La educación primaria abarca 6 cursos académicos (desde los 6 a los 12 ańos de edad) y la secundaria se compone de 4 cursos académicos (desde los 13 hasta los 16 años de edad).
} 
Hay que destacar que, desde el comienzo del estudio, todas las pruebas se realizaron con normalidad y contamos en todo momento con la buena disposición del profesorado del centro. Además, no tuvimos ningún tipo de inconveniente a la hora de evaluar a los alumnos, ya que tanto las familias como el profesorado, estaban muy interesados en conocer el nivel de inglés de sus estudiantes/hijos y, a su vez, saber si existía mucha diferencia de nivel entre los alumnos que siguen enseñanzas bilingües y no bilingües.

\subsection{Prueba de examen: nivel, partes y puntuación del mismo}

El examen realizado por el alumnado es de nivel PET (Preliminary English Test) correspondiente a una prueba de inglés de nivel intermedio que se sitúa en el nivel B1 del Marco Común Europeo de Referencia para las lenguas (MCER). Esta prueba de evaluación fue elaborada en el curso académico 2014/2015 por los asesores de Cambridge Assessment para nuestro estudio. Consta de cuatro partes que cubren las cinco destrezas básicas de aprendizaje de una lengua extranjera: lectura, escritura, escucha, hablar y conversar. Estas cinco partes del examen se presentan en dos formatos el examen escrito (lectura, escritura y escucha) y el examen oral (hablar y conversar). En cuanto a la puntuación, el examen está diseńado sobre 100 puntos: 5 puntos corresponden a la parte de lectura, 15 puntos corresponden a la parte de escritura, 25 puntos a la parte de escucha y, finalmente, otros 25 puntos a la parte de hablar y conversar. A continuación, vamos a detallar cada una de las partes del examen, tanto escrito como oral, y el modo en el que se puntúa cada una de ellas.

\subsubsection{El examen escrito}

El examen escrito consta de tres partes: lectura, escritura y escucha. Las partes de lectura y escritura juntas representan un $50 \%$ de la puntuación del examen total (35 puntos lectura y 15 puntos escritura) y la de escucha representa un $25 \%$. Así, en total, el examen escrito en su totalidad cubre el $75 \%$ de la nota final.

En el apartado de lectura (reading) se mide el nivel de compresión escrita mediante cinco ejercicios en los que se presentan varios textos y sobre ellos se hacen preguntas de elección múltiple, de seleccionar verdadero/falso, de unir o de completar huecos. Cada una de las repuestas correctas corresponde a un punto en el examen. De este modo, la nota máxima en esta actividad serían 35 puntos.

En el apartado de escritura (writing), a su vez, se mide el nivel de expresión escrita a través de tres actividades. En la primera actividad se tiene que escribir una frase similar a la propuesta usando una palabra de referencia. En la segunda actividad los alumnos tienen que escribir una postal corta (de entre 35-45 palabras) y, finalmente, en la tercera parte hay que escribir una redacción más larga (una carta o una historia) de unas 100 palabras aproximadamente. En esta parte los alumnos pueden elegir, entre dos opciones, el tema de la redacción que van a desarrollar. En cada una de estas actividades los estudiantes pueden obtener hasta un máximo de 5 puntos por actividad. De este modo, la nota máxima en esta parte de escritura serían 15 puntos. 
En el apartado de escucha (listening) se mide el nivel de comprensión oral con cuatro tipos de escuchas cuya puntuación se corresponde con el 25\% de la nota, es decir, los alumnos pueden obtener una puntuación máxima de 25 puntos en esta parte (un punto por respuesta correcta). Las dos primeras escuchas son de elección múltiple, la tercera escucha es de completar huecos y en la última escucha los estudiantes deben contestar A o B según crean que lo que escuchan es verdadero o falso. Los alumnos oirán dos veces cada una de las escuchas.

\subsubsection{El examen oral}

El examen oral, por otra parte, evalúa las destrezas de hablar y comunicarse y, como vamos a explicar seguidamente, consta de cuatro partes. La nota máxima de esta parte oral del examen son 25 puntos, por lo que la parte oral de hablar y conversar, al igual que la parte de escuchar, representa un $25 \%$ de la nota final. La parte de hablar y comunicarse se denomina examen oral y mide el nivel de expresión oral por parejas. Se desarrolla en cuatro partes de entre dos y tres minutos de duración cada una; por ello, para llevar a cabo la evaluación de esta destreza se necesitan dos examinadores; uno de ellos solamente escucha y el otro formula las preguntas.

En la primera parte de este examen se pregunta a los alumnos su nombre y apellidos, se les pide que lo deletreen y se les hace una o dos preguntas sobre su vida cotidiana, sus gustos, sus aficiones o su familia. En la segunda parte el examinador describe una situación a los alumnos y estos tienen que decidir algo sobre esa situación o llegar a un acuerdo hablando entre ellos. En la tercera parte del examen cada alumno tiene una foto y la tienen que describir individualmente. Finalmente, en la cuarta y última parte de la prueba oral los estudiantes deben conversar entre ellos sobre las fotos que han descrito anteriormente.

En cuanto al tiempo y lugar de realización de la prueba, para la realización de las partes de lectura y escritura los alumnos contaban con 1 hora y 30 minutos. Para la escucha tenían 35 minutos y para la prueba oral (hablar y comunicarse) se les asignaron entre $10 \mathrm{y}$ 15 minutos por pareja. El lugar seleccionado para la realización del examen fue su centro de enseñanza, siempre fuera del horario lectivo. En los ocho centros seleccionados se realizaron las pruebas en horario de tarde.

\section{ANÁlisis y RESUltados}

Tras haber corregido los 201 exámenes diseñados por Cambridge English vamos a exponer los resultados obtenidos en competencia en comunicación lingüística en inglés, tanto en los centros bilingües como en los centros no bilingües. Los resultados se miden en una escala de 0 a 100 puntos, siendo 100 puntos el resultado más alto y considerándose 50 puntos una calificación de aprobado. A continuación, presentamos los resultados obtenidos en las tablas 1 y 2, junto con un gráfico comparativo (Figura 3) en el que aparece una representación visual de los mismos: 
Tabla no 1: Resultados centros bilingües

\begin{tabular}{|l|c|c|c|c|c|}
\hline $\begin{array}{l}\text { CENTROS } \\
\text { CON } \\
\text { PROGRAMA } \\
\text { BILINGÜE }\end{array}$ & $\begin{array}{l}\text { LECTURA } \\
\text { READING }\end{array}$ & $\begin{array}{l}\text { ESCRITURA } \\
\text { WRITING }\end{array}$ & $\begin{array}{l}\text { ESCUCHA } \\
\text { LISTENING }\end{array}$ & $\begin{array}{l}\text { HABLAR Y } \\
\text { CONVERSAR } \\
\text { SPEAKING } \\
\text { AND TALKING }\end{array}$ & TOTAL \\
\hline BILINGÜE 1 & 57,1 & 47,5 & 49,6 & 66,8 & 55,2 \\
\hline BILINGÜE 2 & 74,9 & 74,5 & 81,1 & 77,1 & 76,9 \\
\hline BILINGÜE 3 & 46,0 & 36,2 & 45,9 & 62,1 & 47,5 \\
\hline BILINGÜE 4 & 43,9 & 48,6 & 46,0 & 55,6 & 48,5 \\
\hline TOTAL & $\mathbf{5 5 , 4}$ & $\mathbf{5 1 , 7}$ & $\mathbf{5 5 , 6}$ & $\mathbf{6 5 , 4}$ & $\mathbf{5 7 , 0}$ \\
\hline
\end{tabular}

Observando la tabla 1 podemos comprobar que en los centros bilingües los alumnos obtienen una media de 55,4 puntos sobre 100 en lectura; en escritura el porcentaje asciende a 51,7 puntos; en escucha 55,6 puntos y, por último, en la destreza de hablar y conversar los resultados alcanzan 65,4 puntos. Con las calificaciones de estas cuatro destrezas hallamos una nota media total del nivel de idioma (inglés) de los alumnos pertenecientes a centros bilingües correspondiente a 57 puntos.

Por otro lado, en cuanto a los resultados obtenidos por los alumnos de los centros no bilingües, podemos observar la tabla 2, expuesta a continuación, en la que se aprecia que en los centros no bilingües los alumnos obtienen una media de 48,1 puntos sobre 100 en lectura; en escritura 37,4 puntos; en escucha 46,6 puntos y en la destreza de hablar y conversar 53,1 puntos. Con las calificaciones de estas cuatro destrezas obtenemos una nota media total del nivel de idioma del alumnado perteneciente a centros no bilingües correspondiente a 46,3 puntos.

Tabla no 2: Resultados de centros sin programa bilingüe.

\begin{tabular}{|l|l|l|l|l|l|}
\hline $\begin{array}{l}\text { CENTROS SIN } \\
\text { PROGRAMA } \\
\text { BILINGÜE }\end{array}$ & $\begin{array}{l}\text { LECTURA } \\
\text { READING }\end{array}$ & $\begin{array}{l}\text { ESCRITURA } \\
\text { WRITING }\end{array}$ & $\begin{array}{l}\text { ESCUCHA } \\
\text { LISTENING }\end{array}$ & $\begin{array}{l}\text { HABLAR Y } \\
\text { CONVERSAR } \\
\text { SPEAKING } \\
\text { AND TALKING }\end{array}$ & TOTAL \\
\hline NO BILINGÜE 1 & 42,5 & 35,3 & 39,3 & 50,3 & 41,8 \\
\hline NO BILINGÜE 2 & 42,6 & 24,4 & 42,0 & 38,4 & 36,8 \\
\hline NO BILINGÜE 3 & 41,6 & 24,3 & 40,8 & 43,0 & 37,4 \\
\hline NO BILINGÜE 4 & $\mathbf{6 6 , 0}$ & $\mathbf{6 5 , 9}$ & $\mathbf{6 4 , 3}$ & 80,9 & $\mathbf{6 9 , 2}$ \\
\hline TOTAL & $\mathbf{4 8 , 1}$ & $\mathbf{3 7 , 4}$ & $\mathbf{4 6 , 6}$ & $\mathbf{5 3 , 1}$ & $\mathbf{4 6 , 3}$ \\
\hline
\end{tabular}


A nivel general, como se aprecia en las tablas 1 y 2 , introducidas anteriormente, y en la figura 3, que exponemos a continuación, los resultados nos indican que los alumnos pertenecientes a centros bilingües superan, en todas las destrezas, a los alumnos que cursan estudios en centros no bilingües. Sin embargo, en contra de nuestras expectativas, la puntuación que presentan entre ellos no difiere mucho. En lectura, la diferencia entre los estudiantes de centros bilingües y no bilingües es de 7,3 puntos. En escritura los resultados encontrados son más dispares, y la diferencia es más elevada ascendiendo a 14,3 puntos. En escucha hemos encontrado un resultado de 9 puntos de diferencia entre ambos tipos de alumnado. En la destreza de hablar y conversar la diferencia obtenida es de 12,3 puntos.

Con estos datos podemos afirmar que las destrezas productivas de escribir y de hablar y conversar son en las que los alumnos bilingües superan, por más de 10 puntos sobre 100, a los no bilingües y, por tanto, puede que sean las destrezas más beneficiadas por los programas bilingües en Castilla-La Mancha. Asimismo, es destacable mencionar que, a nivel general, la diferencia entre el alumnado bilingüe y no bilingüe es de 10,7 puntos sobre 100, ya que la puntuación total obtenida por el alumnado bilingüe es de 57 puntos y la del alumnado no bilingüe es de 46,3 puntos. En el gráfico comparativo que presentamos a continuación podemos visualizar esta diferencia de resultados:

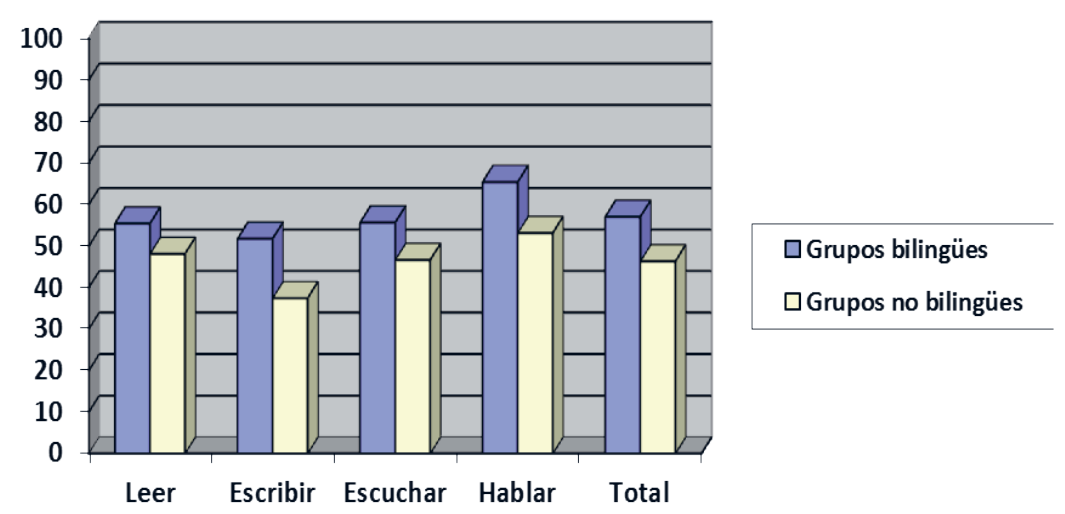

Figura no 3: Resultado por destrezas en $4^{\circ}$ ESO de grupos bilingües y no bilingües.

Debido a que, como acabamos de explicar, los resultados obtenidos en centros bilingües no son tan altos como se esperaba inicialmente, decidimos hacer una comparación de las medias ${ }^{4}$ y las medianas 5 que presentamos en la tabla 3. Estos datos nos serán muy

\footnotetext{
${ }^{4}$ La media aritmética es el valor obtenido al sumar todos los datos y dividir el resultado entre el número total de datos.

${ }^{5}$ La mediana es el valor que ocupa el lugar central de todos los datos cuando éstos están ordenados de menor a mayor.
} 
útiles posteriormente para la realización del test estadístico que vamos a elaborar para comprobar si los resultados obtenidos son realmente significativos. Respecto a las medias, la cifra obtenida en los centros bilingües en lectura es de 5,8406, en escritura es de 5,3741, en escucha de 5,8420, en hablar y conversar asciende a 6,7142 puntos y, la nota media de todas las destrezas en centros bilingües es de 5,9237. En los centros no bilingües las notas medias encontradas son más bajas. En lectura hallamos una nota media de 4,6288, en escritura de 3,5266, en escucha de 4,2568, en hablar y conversar de 5,0797 y, con estos valores, se obtiene una nota media de los centros no bilingües que alcanza 4,3973 puntos. Si comparamos estas notas medias, de nuevo los resultados de centros bilingües superan a los de centros no bilingües con una diferencia de 1,53 puntos en sus notas medias finales.

Tabla no 3: Tabla de medias, medianas y desviación típica ${ }^{6}$.

\begin{tabular}{|l|c|c|c|c|c|c|}
\hline TIPO DE CENTRO & Reading & Writing & Listening & Speaking & Total \\
\hline \multirow{4}{*}{ BILIN } & Media & $\mathbf{5 , 8 4 0 6}$ & $\mathbf{5 , 3 7 4 1}$ & $\mathbf{5 , 8 4 2 0}$ & $\mathbf{6 , 7 1 4 2}$ & $\mathbf{5 , 9 2 3 4}$ \\
\cline { 2 - 7 } & $\mathbf{N}$ & 73 & 73 & 73 & 73 & 73 \\
\cline { 2 - 7 } & Desv. típ. & 2,19020 & 2,73011 & 2,30454 & 1,70771 & 1,99848 \\
\cline { 2 - 7 } & Mediana & $\mathbf{6 , 0 0 0 0}$ & 5,5000 & 5,6000 & 7,2000 & 6,2900 \\
\hline \multirow{4}{*}{ NO BILIN } & Media & $\mathbf{4 , 6 2 8 8}$ & $\mathbf{3 , 5 2 6 6}$ & $\mathbf{4 , 2 5 6 8}$ & $\mathbf{5 , 0 7 9 7}$ & $\mathbf{4 , 3 9 3 7}$ \\
\cline { 2 - 7 } & $\mathbf{N}$ & 128 & 128 & 128 & 128 & 128 \\
\cline { 2 - 7 } & Desv. típ. & 1,90971 & 2,77870 & 2,08839 & 2,86344 & 2,14231 \\
\cline { 2 - 7 } & Mediana & 4,0000 & 3,2500 & 4,0000 & 5,2000 & 4,0450 \\
\hline
\end{tabular}

En cuanto a las medianas, en los centros bilingües los resultados son los siguientes: en lectura la mediana es de 6,0000 puntos, en escritura obtenemos 5,5000 puntos, en escucha 5,6000 puntos y, en hablar y conversar, se alcanzan los 7,200 puntos. Con estas cifras se obtiene una mediana total de 6,2900 puntos en centros bilingües. En los centros no bilingües volvemos a encontrar cifras menores en los valores de las medianas. Así, en lectura la mediana es de 4,000 puntos, en escritura se alcanzan 3,2500 puntos, en escucha 4,0000 puntos y, en hablar y conversar, 5,2000 puntos. Con estas cifras se obtiene una mediana total de 4,0450 puntos en centros no bilingües.

Una vez analizadas las medias y las medianas, y para comprobar si los datos obtenidos en nuestro estudio son significativos o no, vamos a exponer los resultados alcanzados por los

\footnotetext{
${ }^{6}$ Leyenda de la tabla 3: $\mathrm{N}$ es el número de alumnos de centros bilingües y de centros no bilingües; Bilin: calificaciones de alumnos de centros bilingües; No bilin: calificaciones de alumnos de centros no bilingües.
} 
alumnos utilizando el test estadístico SPSS para la comparación de medias de todos los grupos (bilingües y no bilingües). El instrumento que vamos a utilizar para realizar este estudio es el T-Student de muestras independientes, con las calificaciones medias obtenidas por el alumnado bilingüe y no bilingüe. Como podemos apreciar en la tabla 4, la comparación de las notas medias obtenidas nos permite afirmar que los alumnos que asisten a centros bilingües obtienen resultados en todas las destrezas superiores a las medias de los estudiantes que asisten a centros no bilingües con un $\mathrm{p}=0.05$. La significación, o el nivel de certeza que tenemos en nuestra afirmación, nos la otorga el p-valor o p-value. En realidad, esta cifra revela la diferencia entre 1 y el p-valor. Como este test está hecho con un p-valor de 0,05 , los resultados obtenidos nos indican que la certeza con la que hacemos la afirmación de comparación de medias es el 0,95 , o $95 \% 7$. No obstante, esas diferencias no son muy elevadas. En todas las destrezas la media oscila entre 1,2 puntos y 1,8 puntos, lo que supondría una diferencia entre alumnado bilingüe y no bilingüe de entre un $12 \%$ y un $18 \%$. La media del examen total asciende a 1,5 puntos, es decir, existe un $15 \%$ de diferencia entre un tipo de alumnado y el otro.

Tabla no 4: T-test de muestras independientes de las calificaciones medias obtenidas por el alumnado bilingüe y no bilingüe.

\begin{tabular}{|c|c|c|c|c|c|c|c|c|c|c|}
\hline & & \multicolumn{2}{|c|}{$\begin{array}{l}\text { Prueba de } \\
\text { Levene para } \\
\text { la igualdad de } \\
\text { varianzas }\end{array}$} & \multicolumn{7}{|c|}{ Prueba $T$ para la igualdad de medias } \\
\hline & & \multirow[t]{2}{*}{$\mathbf{F}$} & \multirow[t]{2}{*}{ Sig. } & \multirow[t]{2}{*}{$\mathbf{t}$} & \multirow[t]{2}{*}{ gl } & \multirow[t]{2}{*}{$\begin{array}{c}\text { Sig } \\
\text { (bilateral) }\end{array}$} & \multirow[t]{2}{*}{$\begin{array}{l}\text { Diferencia } \\
\text { de medias }\end{array}$} & \multirow[t]{2}{*}{$\begin{array}{c}\text { Error } \\
\text { típ. de la } \\
\text { diferencia }\end{array}$} & \multicolumn{2}{|c|}{$\begin{array}{c}99 \% \text { Intervalo de } \\
\text { confianza para la } \\
\text { diferencia }\end{array}$} \\
\hline & & & & & & & & & Inferior & Superior \\
\hline \multirow{2}{*}{ Reading } & \begin{tabular}{|l} 
Se han \\
asumido \\
varianzas \\
iguales \\
\end{tabular} & 3,143 &, 078 & 4,099 & 199 &, 000 & 1,21185 & ,29564 & ,44297 & 1,98073 \\
\hline & $\begin{array}{l}\text { No se han } \\
\text { asumido } \\
\text { varianzas } \\
\text { iguales }\end{array}$ & & & 3,948 & 133,720 &, 000 & 1,21185 & ,3069 & ,40982 & 2,01388 \\
\hline \multirow{2}{*}{ Writing } & \begin{tabular}{|l} 
Se han \\
asumido \\
varianzas \\
iguales
\end{tabular} &, 274 & ,601 & 4,562 & 199 &, 000 & 1,84755 & ,40498 & ,79429 & 2,90080 \\
\hline & $\begin{array}{l}\text { No se han } \\
\text { asumido } \\
\text { varianzas } \\
\text { iguales }\end{array}$ & & & 4,584 & 152,106 &, 000 & 1,84755 & ,40302 & ,79626 & 2,89884 \\
\hline
\end{tabular}

\footnotetext{
${ }^{7}$ Téngase en cuenta que nunca puede ser una certeza del $100 \%$ porque eso sería una verdad absoluta, hecho que no existe en probabilidad.
} 


\begin{tabular}{|l|l|l|l|l|l|l|l|l|l|l|}
\hline Listening & $\begin{array}{l}\text { Se han } \\
\text { asumido } \\
\text { varianzas } \\
\text { iguales }\end{array}$ \\
\begin{tabular}{|l} 
Speaking \\
$\begin{array}{l}\text { No se han } \\
\text { asumido } \\
\text { varianzas } \\
\text { iguales }\end{array}$
\end{tabular} & 1,678 &, 197 & 4,983 & 199 &, 000 & 1,58524 &, 31813 &, 75785 & 2,41262 \\
\hline $\begin{array}{l}\text { Se han } \\
\text { asumido } \\
\text { varianzas } \\
\text { iguales }\end{array}$ & 23,182 &, 000 & 4,444 & 199 &, 000 & 1,63456 &, 36777 &, 67806 & 2,59105 \\
\hline $\begin{array}{l}\text { No se han } \\
\text { asumido } \\
\text { varianzas } \\
\text { iguales }\end{array}$ \\
Total
\end{tabular}

Con estos resultados podemos afirmar que las diferencias entre estudiantes pertenecientes a centros bilingües y a no bilingües, aunque estadísticamente significativas, alcanzan solamente una diferencia de 1,5 puntos en una escala de 1 a 10. Esto es, en principio, una puntuación baja si tenemos en cuenta, por un lado, el elevado número de horas que el alumnado bilingüe está expuesto a esta lengua extranjera y, por otro, los resultados obtenidos en otras comunidades autónomas que, como mostraremos en la sección 4 de este artículo, son superiores a los obtenidos por los alumnos de la comunidad castellano-manchega.

\section{Comparación de los resultados obtenidos en la comunidad de Castilla-La Mancha CON OTRAS COMUNIDADES AUTÓNOMAS ESPAŃOLAS}

Para comparar los resultados estadísticos de nuestro estudio con los alcanzados en otras comunidades autónomas hemos buscado zonas que comenzaron sus proyectos bilingües al mismo tiempo que en Castilla-La Mancha y, por lo tanto, tienen los mismos ańos de experiencia en educación bilingüe. Así, en el contexto andaluz, un estudio realizado por Lorenzo et al. en el año 2009 con 1320 alumnos de primaria y secundaria, con 754 alumnos escolarizados en programas bilingües y 448 del grupo de control no bilingüe, puso de manifiesto el impacto positivo en los niveles competenciales tanto en la lengua materna como en la lengua extranjera de los alumnos pertenecientes a las secciones bilingües. De este modo quedó patente que los alumnos de programas lingüísticos consiguieron en las pruebas de lengua extranjera una puntuación que era un $24 \%$ más de media que la del grupo de control no bilingüe (Travé 2013: 382). Esta diferencia de nivel es notablemente 
superior a la que nosotros hemos encontrado en Castilla-La Mancha, donde los alumnos de centros bilingües alcanzan una media superior a la de los alumnos no bilingües en un 15\% únicamente. Con estos datos comprobamos que en Andalucía los resultados hallados entre alumnado bilingüe y no bilingüe son un $9 \%$ superiores a los de Castilla-La Mancha.

A su vez, en el País Vasco, Lasagabaster comparó 198 estudiantes pertenecientes a programas de estudios bilingües y no bilingües en educación secundaria en cuatro institutos diferentes (Lasagabaster 2008). Todos ellos empezaron a aprender inglés como lengua extranjera a los 8 años. Los resultados obtenidos indicaban que los estudiantes bilingües superaban en todas las destrezas a los no bilingües con una diferencia media del $40 \%$. Con estos resultados se afirmaba que la metodología AICLE tenía un claro impacto en la adquisición de la lengua extranjera. Este resultado que muestra David Lasagabaster en su investigación contrasta con el leve o menor impacto que hemos hallado nosotros en nuestro estudio en Castilla-La Mancha (recordemos que solamente hay un 15\% de diferencia). Ahora bien, tenemos que tener en cuenta que esta comunidad ya tiene experiencia previa en enseñanzas bilingües espańol-vasco y puede ser uno de los motivos principales por los cuales sus resultados son tan elevados y positivos.

En Cataluña existen otras investigaciones como las de Navés \& Victori (2010) que, aunque no aportan datos estadísticos, también prueban que los estudiantes que siguen programas bilingües en octavo curso superan en nivel de idioma en todas las destrezas a estudiantes que no los siguen de un curso superior (noveno curso) (Sylvén 2013: 301). Con esta información volvemos a poner de manifiesto el impacto beneficioso de la metodología AICLE en el aprendizaje de la lengua extranjera.

Respecto al número de alumnos que han aprobado el examen diseñado por Cambridge Assessment para nuestro estudio, hemos obtenido un 64,38 \% de alumnos aprobados en centros bilingües y un $35,15 \%$ en centros no bilingües de esta región. Con estos datos confirmamos que casi el doble del alumnado de centros bilingües ha aprobado el examen en Castilla-La Mancha. Estos resultados son comparables con los obtenidos en Galicia, donde el $87,7 \%$ de los alumnos de centros bilingües aprueban, frente a un $45,1 \%$ de alumnado que aprueba el examen en centros no bilingües. De nuevo, en ambas comunidades, casi el doble de alumnos de centros bilingües aprueba el examen que se propuso a los alumnos para testar su nivel de competencia lingüística en lengua inglesa.

En el País Vasco también se encuentra un número superior de alumnos que aprueba el examen de competencia en comunicación lingüística en lengua inglesa, pero los resultados obtenidos en esta comunidad no son tan proporcionales. En el estudio de Grisaleña et al., realizado en el primer ciclo de educación secundaria ( $1^{\circ}$ y $2^{\circ}$ de ESO), el $86,96 \%$ de los alumnos bilingües aprueban el examen de nivel A2 del Marco Común Europeo de Referencia de las Lenguas (MCER), frente a un 31,58\% de los alumnos no bilingües (Grisaleña et al. 2009). En este sentido, más del doble de los alumnos bilingües aprueban el examen, pero tenemos que destacar que el nivel educativo evaluado es un ciclo inferior al de nuestro estudio, realizado en Castilla-La Mancha en el segundo ciclo de Educación Secundaria Obligatoria. 
En el mismo estudio de Grisaleña et al., pero realizado a estudiantes del segundo ciclo de Educación Secundaria, con un examen de nivel B1 del MCER (mismo nivel que en nuestro estudio), el número de alumnos bilingües aprobados fue del 78,57\%, frente al $15 \%$ de alumnos no bilingües que aprobaron la misma prueba. En este caso, el número de alumnos bilingües que aprueba el examen de nivel B1 quintuplica al número de alumnos no bilingües. En la tabla 5 podemos apreciar los resultados de nuestro estudio y comparar el porcentaje de alumnos aprobados en Castilla-La Mancha con el de alumnos aprobados en otras comunidades autónomas como Galicia y el País Vasco:

Tabla no 5: Comparación del porcentaje de estudiantes aprobados en Castilla-La Mancha, Galicia y País Vasco.

\begin{tabular}{|l|c|c|c|c|}
\hline & $\begin{array}{l}\text { APROBADOS } \\
\text { EN CASTILLA } \\
\text { LA MANCHA }\end{array}$ & $\begin{array}{l}\text { APROBADOS } \\
\text { EN GALICIA }\end{array}$ & $\begin{array}{l}\text { APROBADO } \\
\text { EN EL PAÍS } \\
\text { VASCO }\end{array}$ & $\begin{array}{l}\text { APROBADO } \\
\text { EN EL PAÍS } \\
\text { VASCO }\end{array}$ \\
\hline CURSO EVALUADO & $4^{\circ}$ ESO & $4^{\circ}$ ESO & $1^{\circ}$ Y $2^{\circ}$ ESO & $3^{\circ}$ Y $4^{\circ}$ ESO \\
\hline BILINGUE & $64,38 \%$ & $87,7 \%$ & $86,96 \%$ & $78,57 \%$ \\
\hline NO BILINGUE & $35,15 \%$ & $45,1 \%$ & $31,58 \%$ & $15 \%$ \\
\hline
\end{tabular}

Tanto en la tabla 5 que acabamos de exponer como en la figura 4 que presentamos seguidamente podemos observar que el porcentaje de alumnos bilingües y no bilingües que aprueban el examen de lengua extranjera (inglés) es superior en alumnos bilingües frente a los no bilingües en todos los estudios. A su vez, también es destacable que el porcentaje de alumnos bilingües aprobados más bajo pertenece a nuestro estudio en Castilla-La Mancha. A este respecto, podemos comentar que tanto Galicia como el País Vasco son comunidades bilingües en gallego-español y vasco-español, respectivamente. De esta forma, los alumnos de estas comunidades autónomas ya están habituados y tienen más facilidad y experiencia en enseñanzas bilingües y, por lo tanto, en el uso de la metodología AICLE. No obstante, aunque esta puede ser la causa fundamental que determina las diferencias existentes entre la Comunidad Autónoma de Castilla-La Mancha y otras comunidades del territorio español, consideramos que tenemos que seguir indagando para buscar otras causas que puedan influir en los mejores resultados obtenidos por alumnos que cursan programas bilingües en otras comunidades autónomas. 


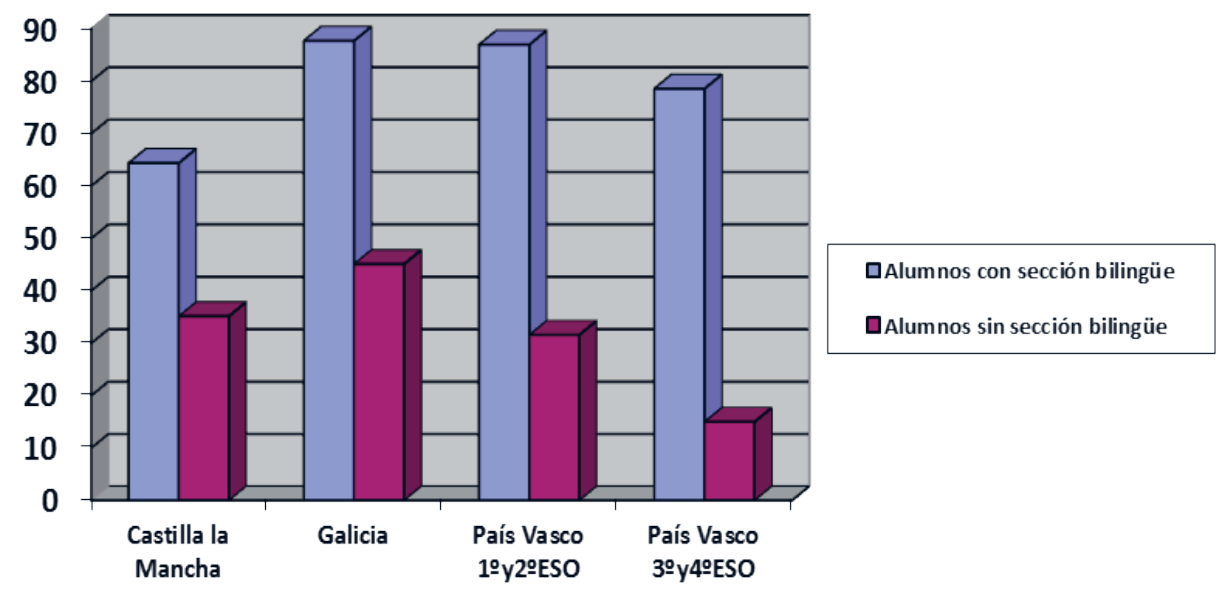

Figura 4: Comparación de alumnos aprobados en Castilla-La Mancha y Galicia.

\section{Conclusiones}

Al comienzo de esta investigación nos propusimos demostrar si los alumnos de $4^{\circ}$ curso de ESO, pertenecientes a programas bilingües ofertados por centros educativos públicos en la comunidad autónoma de Castilla-La Mancha, obtenían mejores resultados globales en la evaluación de la competencia comunicativa en lengua inglesa que los alumnos involucrados en contextos no bilingües. A su vez, también nos planteamos comparar estos resultados con los de otras Comunidades Autónomas Españolas. Por ello, una vez realizado el análisis y establecidas las comparaciones entre los resultados en competencia lingüística conseguidos por alumnos de programas bilingües y no bilingües de Castilla-La Mancha y de otras Comunidades Autónomas Españolas, podemos concluir afirmando que los resultados de este estudio nos indican que en Castilla-La Mancha, al igual que en el resto de estudios realizados en Andalucía, el País Vasco, Galicia o Cataluña mencionados previamente, los alumnos pertenecientes a centros bilingües superan, en todas las destrezas, a los resultados obtenidos por los alumnos de centros no bilingües. En cuanto a las destrezas comunicativas se refiere, es importante mencionar que los alumnos pertenecientes a centros bilingües de Castilla-La Mancha aprueban todas las destrezas evaluadas en la prueba de competencia lingüística, pero con puntuaciones bajas, siendo 65,4 puntos sobre 100 la máxima nota obtenida en expresión oral. Por el contrario, los alumnos pertenecientes a centros no bilingües de esta misma comunidad autónoma sólo aprueban una destreza, la expresión oral: hablar y conversar, consiguiendo una puntuación media de 53,1 puntos sobre 100 .

Como hemos podido comprobar, la destreza en la que obtienen una mayor puntuación los alumnos de los centros bilingües y no-bilingües castellano-manchegos es la expresión oral (hablar y conversar). Esto puede indicar que, en ambos centros de enseńanza 
se está siguiendo la misma metodología comunicativa y de fomento de participación oral del alumnado en el aula para el desarrollo de esta habilidad. Por este motivo los alumnos se sienten más seguros de sí mismos al hablar y conversar en lengua extranjera, frente a otras destrezas, quizás menos practicadas en los centros no bilingües.

De la conclusión anterior comprobamos que en Castilla-La Mancha hay diferencias importantes respecto a otros estudios de otras comunidades autónomas en España (Lasagabaster y Ruiz de Zarobe 2010, Llinares y Whittaker 2009, Dafouz y Guerrini 2009) y en Europa (Swain 1995, Bialystok 2005, Dalton-Puffer 2007-2008), en los que los estudiantes AICLE obtienen mejores calificaciones en las destrezas receptivas de comprensión oral y escrita (escuchar y leer). Por el contrario, en Castilla-La Mancha se produce el efecto contrario y los alumnos AICLE muestran un mayor desarrollo, en concreto, en una destreza productiva: la expresión oral (hablar y conversar), destreza que, a su vez, es también la más beneficiada por las enseñanzas no bilingües en el territorio castellano-manchego.

En relación a la comprensión escrita (leer) y la comprensión oral (escuchar), no encontramos diferencias notables entre centros bilingües y no bilingües en CastillaLa Mancha. Aunque los resultados obtenidos por los alumnos de centros bilingües son superiores, los datos indican que la diferencia respecto a los resultados conseguidos por alumnos de centros no bilingües es poco elevada, ascendiendo estadísticamente en 1,2 puntos en comprensión escrita y 1,6 puntos en comprensión oral.

Respecto a la expresión oral (hablar y conversar) y la expresión escrita (escribir) los resultados nos indican que hay diferencias notables entre centros bilingües y no bilingües en Castilla-La Mancha, sobre todo en expresión escrita (escribir). Estadísticamente en expresión oral los alumnos bilingües superan en 1,6 puntos a los alumnos no bilingües. En cuanto a la expresión escrita, los alumnos bilingües superan en 1,8 puntos a los alumnos de programas no bilingües. Estas diferencias entre alumnos bilingües y no bilingües se pueden deber a varios motivos. Por un lado, en cuanto a la expresión oral se refiere, podemos señalar que la metodología AICLE fomenta la comunicación y la interacción en el aula y, por ello, se aprecia una mejora del alumnado AICLE respecto al no AICLE aunque, como hemos mencionado anteriormente, esta es la destreza que mejor dominan los alumnos no bilingües. Por otro, con referencia a la expresión escrita, tenemos que tener en cuenta que este estudio se ha llevado a cabo en el último curso de Educación Secundaria y que la competencia escrita general se adquiere a largo plazo, dependiendo más de la madurez del alumno que del tipo de instrucción recibida en lengua extranjera (Dalton-Puffer 2011: 187). Así, podemos afirmar que las calificaciones de alumnos bilingües en expresión escrita son superiores a las de alumnos no bilingües. Esto se puede deber, posiblemente, a la combinación de varios factores: el uso de la metodología AICLE y la madurez del alumno perteneciente a $4^{\circ}$ curso de ESO.

Acerca del número de alumnos que han aprobado el examen, debemos mencionar que en los centros bilingües el número de aprobados $(64,38 \%)$ es superior al de alumnos pertenecientes a centros no bilingües (35,15\%). Sin embargo, aunque el número de alumnos 
aprobados es casi el doble en alumnos bilingües que en no bilingües, la puntuación obtenida por los alumnos bilingües es, como hemos indicado anteriormente, baja. Como señalan Serrano \& Muñoz, esta baja puntuación obtenida por los alumnos de centros bilingües confirma que el hecho de que exponer al alumnado a un elevado número de horas en otra lengua no asegura un mayor y eficaz aprendizaje de la misma (Serrano \& Muñoz 2007).

Con este análisis realizado en las aulas de Castilla-La Mancha podemos afirmar que, aunque se aprecia un incremento en el nivel de idioma extranjero en los alumnos que siguen enseñanzas bilingües, se necesita una mejora en la implantación del programa de educación bilingüe. Sería deseable que los alumnos que cursan este tipo de programas en lengua extranjera obtuvieran mejores resultados y mucho más elevados tras más de ocho años siguiendo la metodología AICLE y habiendo estado expuestos al triple de clases impartidas en lengua inglesa que los alumnos pertenecientes a enseñanzas tradicionales. Esto es un reto que la comunidad castellano-manchega debe afrontar en un futuro inmediato. Como indica Goodman, intentar hacer un país bilingüe, o en nuestro caso, una región bilingüe, no es una tarea fácil (Goodman 2007: 232).

\section{Obras Citadas}

Admiraal, Wilfried, Gerard Westhoff \& Kees de Bot. 2006. "Evaluation of bilingual secondary education in the Netherlands: Students' language Proficiency in English". Educational Research and Evaluation 12: 75-93.

Bialystok, Ellen. 2005. "Consequences of bilingualism for cognitive development". En: J. Kroll, \& A. De Groot. Eds. Handbook of bilingualism: psycholinguistic approaches. Oxford: Oxford University Press. 27-48.

Bruton, Anthony. 2011. "Are the differences between CLIL and non-CLIL groups in Andalusia due to CLIL? A reply to Lorenzo, Casal and Moore". Applied Linguistics 32: 236-241.

Dalton-Puffer, Christiane. 2007. Discourse in Content and Language Integrated Learning (CLIL). Amsterdam-Philadelphia: John Benjamins Publishing Company.

Dalton-Puffer, Christiane. 2008. "Outcomes and processes in Content and Language Integrated Learning (CLIL): current research from Europe". En: W. Delanoy y L. Volkmann. Eds. Future Perspectives for English Language Teaching. Heidelberg: Carl Winter. 139-157.

Dalton-Puffer, Christiane. 2011. "Content and Language Integrated learning: From practice to principle?" Annual Review of Applied Linguistics 31: 182-204.

García, Ofelia. 2009. Bilingual Education in the 21st Century: A Global Perspective. Chicester: Wiley-Blackwell.

Goodman, David. 2007. "Performance Phenomena in Simultaneous and Sequential Bilinguals: a Case Study of Two Chilean Bilingual Children". Revista electrónica de Literatura y Lingüistica UACH 18: 219-232. 
Grisaleña, Jesús, Alejandro Campo \& Esmeralda Alonso. 2009. "Enseñanza plurilingüe en centros de educación secundaria: análisis de resultados". Revista lberoamericana de Educación 49 (1): 1-12.

Guerrini, Michele. 2009. "CLIL Materials as Scaffolds to Learning". En: D. Marsh, P. Mehisto, D. Wolff, R. Aliaga, T. Asikainen, M.J. Frigols-Martin, S. Hughes \& G. Langé. Eds. CLIL Practice: Perspectives from the Field. Finland: University of Jyväskylä. 74-84.

Jiménez Catalán, Rosa María \& Yolanda Ruiz de Zarobe. 2009. “The Receptive Vocabulary of EFL Learners in Two Instructional Contexts: CLIL versus non-CLIL Instruction”. En: Y. Ruiz de Zarobe \& R.M. Jiménez Catalán. Eds. Content and Language Integrated Learning. Evidence from Research in Europe. Bristol: Multilingual Matters. 81-92.

Lasagabaster, David. 2008. "Foreign language competence in content and language integrated learning". Open Applied Linguistics Journal 1: 31-42.

Lasagabaster, David \& Yolanda Ruiz de Zarobe. 2010. CLIL in Spain. Implementations, Results and Teacher Training. Newcastle: Cambridge Scholars Publishing.

Loranc-Paszylk, Barbara. 2009. "Integrating Reading and Writing into the Context of CLIL Classroom: Some Practical Solutions”. International CLIL Research Journal 1 (2): 46- 53 .

Lorenzo, Francisco. 2010. "CLIL in Andalusia”. En: D. Lasagabaster \& Y. Ruiz de Zarobe. Eds. CLIL in Spain: Implementation, Results and Teacher Training. Newcastle: Cambridge Scholars Publishers. 2-11.

Lorenzo, Francisco, Sonia Casal \& Pat Moore. 2009. “The Effects of Content and Language Integrated Learning in European Education: Key Findings from the Andalusian Bilingual Sections Evaluation Project”. Applied Linguistics 31 (3): 418-442.

Mehisto, Peter, David Marsh \& Ma Jesús Frigols. 2008. Uncovering CLIL. Content and Language Integrated Learning in Bilingual and Multilingual Education. Oxford: Macmillan.

Navés, Teresa. 2011. "How Promising Are the Results of Integrating Content and Language for EFL Writing and Overall EFL Proficiency?”. En: Ruiz de Zarobe, Y., Sierra, J. \& Gallardo del Puerto, F. Eds. Content and Foreign Language Integrated Learning. Bern: Peter Lang. 103-128.

Navés, Teresa \& Mia Victori. 2010. "CLIL in Catalonia: An Overview of Research Studies”. En: Y. Ruiz de Zarobe \& D. Lasagabaster. Eds. CLIL in Spain: Implementation, results and teacher training. Newcastle: Cambridge Scholars. 30-54.

Salis, Edward. 2002. Total quality management in education. London: Routledge.

San Isidro, Xavier. 2009. "Galicia: Clil Success in a Bilingual Community”. En: D. Marsh, P. Mehisto, D. Wolff, R. Aliaga, T. Asikainen, M.J Frigols-Martin, S. Hughes \& G. Langé. Eds. CLIL Practice: Perspectives from the Field. Jyväskylä: University of Jyväskylä. 4-13.

San Isidro, Xavier. 2010. “An Insight into Galician CLIL: Provision and Results”. En: D. Lasagabaster \& Y. Ruiz de Zarobe. Eds. CLIL in Spain: Implementation, results and 
teacher training. Newcastle: Cambridge Scholars. 55-78.

Swain, Merrill. 1995. "Three functions of output in second language learning". En: G. Cook \& B. Seidlhofer. Eds. Principles and practice in the study of language. Oxford: Oxford University Press. 140-142.

Sylvén, Liss Krestin. 2013. "CLIL in Sweden- Why does it not work? A metaperspective on CLIL across contexts in Europe". International Journal of Bilingual Education and Bilingualism 16 (3): 301-320.

Travé González, Gabriel. 2013. "Un estudio sobre las representaciones del profesorado en Educación Primaria acerca de la enseñanza bilingüe”. Revista de educación 361: 379402.

Van de Craen, Piet; Katrien Mondt; Laure Allain \& Ying Gao. 2007. "Why and How CLIL Works. An Outline for a CLIL Theory". VIEWS Vienna English Working Papers 18 (3): 70- 78.

Whittaker, Rachel \& Ana Llinares. 2009. "CLIL in Social Science Classrooms: Analysis of Spoken and Written Productions”. En: Y. Ruiz de Zarobe \& R. M. Jiménez Catalán. Eds. Content and Language Integrated Learning. Evidence from research in Europe. Bristol: Multilingual Matters. 215-234. 